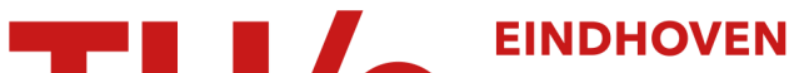

\section{Capacitive current interruption with air-break high voltage disconnectors}

\section{Citation for published version (APA):}

Chai, Y., Wouters, P. A. A. F., Hoppe, van, R. T. W. J., Smeets, R. P. P., \& Peelo, D. F. (2010). Capacitive current interruption with air-break high voltage disconnectors. IEEE Transactions on Power Delivery, 25(2), $762-$ 769. https://doi.org/10.1109/TPWRD.2009.2034746

DOI:

10.1109/TPWRD.2009.2034746

Document status and date:

Published: 01/01/2010

\section{Document Version:}

Publisher's PDF, also known as Version of Record (includes final page, issue and volume numbers)

\section{Please check the document version of this publication:}

- A submitted manuscript is the version of the article upon submission and before peer-review. There can be important differences between the submitted version and the official published version of record. People interested in the research are advised to contact the author for the final version of the publication, or visit the $\mathrm{DOI}$ to the publisher's website.

- The final author version and the galley proof are versions of the publication after peer review.

- The final published version features the final layout of the paper including the volume, issue and page numbers.

Link to publication

\section{General rights}

Copyright and moral rights for the publications made accessible in the public portal are retained by the authors and/or other copyright owners and it is a condition of accessing publications that users recognise and abide by the legal requirements associated with these rights.

- Users may download and print one copy of any publication from the public portal for the purpose of private study or research.

- You may not further distribute the material or use it for any profit-making activity or commercial gain

- You may freely distribute the URL identifying the publication in the public portal.

If the publication is distributed under the terms of Article $25 \mathrm{fa}$ of the Dutch Copyright Act, indicated by the "Taverne" license above, please follow below link for the End User Agreement:

www.tue.nl/taverne

Take down policy

If you believe that this document breaches copyright please contact us at:

openaccess@tue.nl

providing details and we will investigate your claim. 


\title{
Capacitive Current Interruption With Air-Break High Voltage Disconnectors
}

\author{
Yajing Chai, P. A. A. F. Wouters, R. T. W. J. van Hoppe, R. P. P. Smeets, Fellow, IEEE, and
}

D. F. Peelo, Senior Member, IEEE

\begin{abstract}
Capacitive current interruption with air-break disconnectors in a high-voltage network is an interactive event between the circuit and arc with a variety of interruptions and reignitions. In this contribution, first, a theoretical analysis related to this interaction is presented. The effect of capacitances at the source side $\left(C_{s}\right)$ and load side $\left(C_{l}\right)$ is investigated. Three distinct frequencies are identified as contributing to the voltage and current events in the circuit. Besides the power frequency quantities, a medium frequency transient arises related to the excursion of voltage across capacitances to the applied voltage, and a high-frequency transient arises due to charge redistribution between load- and source-side capacitance at reignition. Second, experimental results from an interruption measurement are studied in detail. Typical waveshapes of voltages across the capacitances, disconnector, and currents through the disconnector show that the transients during interrupted are in agreement with the theoretical analysis. Reignition voltage of the air gap and energy input to the arc on reignition are also studied. It is concluded that besides a higher interruption current and a higher power supply level, a lower $C_{s} / C_{l}$ ratio leads to more severe interruption and longer arc duration. Finally, the actual status of IEC recommendations on testing, that has taken into account this arc-circuit interaction, is summarized.
\end{abstract}

Index Terms-Arc, capacitive current, disconnector, disconnect switches, high voltage, interruption, measurements, reignition, standards, substation, testing.

\section{NOMENCLATURE}

$\begin{array}{ll}R_{s}, L_{s} & \text { Inductance and resistance at the source. } \\ C_{s}, C_{l} & \text { Capacitance at the source and load side. } \\ i_{d} & \text { Current through the disconnector. } \\ u_{s} & \text { Voltage of the power supply of the network. } \\ U_{s} & \text { Value of } u_{s} \text { at } t=0 . \\ E_{m} & \text { Amplitude of } u_{s} .\end{array}$

Manuscript received November 18, 2008; revised July 01, 2009, August 25, 2009. Current version published March 24, 2010. Paper no. TPWRD-008612008.

Y. Chai, P. A. A. F. Wouters, and R. T. W. J. van Hoppe are with the Department of Electrical Engineering, Electrical Power Systems Group, Eindhoven University of Technology, Eindhoven $5600 \mathrm{MB}$, the Netherlands. (e-mail: y.chai@tue.nl; p.a.a.f.wouters@tue.nl; r.t.w.j.v.hoppe@tue.nl)

R. P. P. Smeets is with the Department of Electrical Engineering, Electrical Power Systems Group, Eindhoven University of Technology, Eindhoven $5600 \mathrm{MB}$, The Netherlands. He is also with the KEMA T\&D Testing Services, Arhnem 6812 AR, The Netherlands (e-mail: rene.smeets@kema.com).

D. F. Peelo is with D. F. Peelo and Associates, Surrey, BC V4A 2C7, Canada (e-mail: dfpeelo@ieee.org).

Color versions of one or more of the figures in this paper are available online at http://ieeexplore.ieee.org.

Digital Object Identifier 10.1109/TPWRD.2009.2034746

$\begin{array}{ll}\omega_{p} & \text { Angular power frequency of } u_{s} . \\ \phi & \text { Initial phase angle of } u_{s} . \\ u_{c p} & \text { Voltage across the capacitances at power } \\ & \text { frequency. } \\ u_{c s}, u_{c l} & \text { Voltage across } C_{s} \text { and } C_{l} . \\ u_{d} & \text { Voltage across disconnector. } \\ u_{r} & \text { Reignition voltage of the air gap. } \\ \mathrm{HF}, \mathrm{MF}, \mathrm{PF} & \text { High, medium, power frequency. } \\ U_{E} & \text { Equalization voltage at HF oscillation. } \\ R_{H}, L_{H} & \text { Equivalent resistance, inductance of HF } \\ \omega_{0 H}, \omega_{0 M} & \text { loop. } \\ \beta_{H}, \beta_{M} & \text { Resonance frequency at HF and MF. } \\ E_{\text {arc }} & \text { Initial phase angle of HF and MF oscillation. } \\ i_{H}, i_{M} & \text { Energy dissipation. } \\ u_{C M} & \text { Current through disconnector at HF and MF. } \\ U_{r} & \text { Voltage across } C_{s} \text { and } C_{l} \text { at MF. } \\ U_{c s}, U_{c l} & \text { Reignition voltage at } t=0 . \\ C_{H} & \text { Voltage across } C_{s} \text { and } C_{l} \text { at } t=0 . \\ \delta_{H}, \delta_{M} & \text { Capacitance of } C_{s}, C_{l} \text { in series. } \\ u_{c l}^{\prime} & \text { Damping constant at HF, MF. } \\ & \end{array}$

\section{INTRODUCTION}

$\mathbf{I}$ $\mathrm{N}$ a power substation, disconnectors (in North America, disconnectors are called disconnect switches) are commonly used mechanical devices. The definition of a disconnector is: "A mechanical switching device which provides, in open position, an isolating distance in accordance with specific requirements" by the International Electrotechnical Vocabulary (IEV) 441-14-05. That means disconnectors only have a safety function. However, in practice due to parasitic capacitances such as from unloaded bus bars, lines etc. in the networks, there is always a capacitive current that disconnectors need to interrupt. Moreover, although not designed for interrupting current, disconnectors do have a certain current interrupting capability thanks to one or more moving contacts during switching operations. According to the IEC 62271-102 [1], this small capacitive current, which is called "negligible current", does not exceed 0.5 A for rated voltage $420 \mathrm{kV}$ and below. In the past, the current 
interrupting capability of the air-break disconnectors has been therefore taken as $0.5 \mathrm{~A}$ or less. Nowadays, with the fast development of power networks in the world, user's requirement for small capacitive current interruption using air-break disconnectors frequently exceeds the above stated $0.5 \mathrm{~A}$.

Literature related to capacitive current interruption using airbreak disconnectors is sparse, for instance [2]-[15]. A good overview is provided by [12]. The principal work in the past is that from F. E. Andrews in the 1940s. Some results from literature such as [3], [8] were collected for IEC and IEEE recommendations [11]. However, the literatures provide only a limited insight into the mechanism of the capacitive current interruption by an air-break disconnector. In this contribution we will therefore present a more detailed approach to this electrical phenomenon during arcing that, by the associated voltage and current transients, may endanger nearby network components such as (instrument) transformers.

Specifically, a study both on theory and experiment is presented in detail. In principle, the capacitive current interruption capability of a disconnector may be affected by various factors such as air humidity, wind speed, earthing type of the system and phase spacing. In this paper, however, only effects of electrical parameters, such as capacitances, inductances, etc. are evaluated. First, a theoretical analysis for steady and transient state phenomena through high-, medium-, and power frequency is given. Second, based on measured data, factors affecting the arc characteristics, reignition voltages and other features such as energy input into the arc on reignition and transient recovery voltage are analyzed and the results are discussed in detail. Finally, conclusions and suggestions for standardization are given.

\section{Theoretical AnAlysis of CAPACITIVE CURRENT INTERRUPTION}

Capacitive current interruption with a disconnector consists of a succession of interactive events between circuit and arc with a repetitive sequence of interruptions and reignitions. The reignition is characterized in terms of oscillation frequency, transients of current and voltage, etc. An arc is characterized in terms of arc duration, arc reach (perpendicular distance of outermost arc position to a line connecting the contacts), arc type (repetitive or continuous), energy input into arc from circuit during the reignition, and so forth.

The basic equivalent circuit for capacitive current interruption is shown in Fig. 1. The disconnector is marked with $D$; The short-circuit inductance $L_{s}$ is based on the short-time current for which the disconnector is rated; $R_{s}, C_{s}$ and $C_{l}$ stand for the resistance, capacitance at the source- and load side, respectively; $i_{d}$ is the current through the disconnector to be interrupted; $u_{s}$ is the voltage of the power supply of the network.

Before the interruption starts, the disconnector is closed. The entire circuit of Fig. 1 is energized: $u_{s}=E_{m} \sin \left(\omega_{p} t+\phi\right)$ with $\omega_{p}$ the angular power frequency, $E_{m}$ the amplitude, $\phi$ the initial phase angle. The current $i_{d}$ and the voltage across the (parallel) capacitances, denoted as $u_{c p}$ are

$$
\left\{\begin{array}{l}
u_{c p}=E_{m} \sin \left(\omega_{P} t+\phi\right) \\
i_{d}=C_{l} \omega_{P} E_{m} \cos \left(\omega_{P} t+\phi\right)
\end{array} .\right.
$$

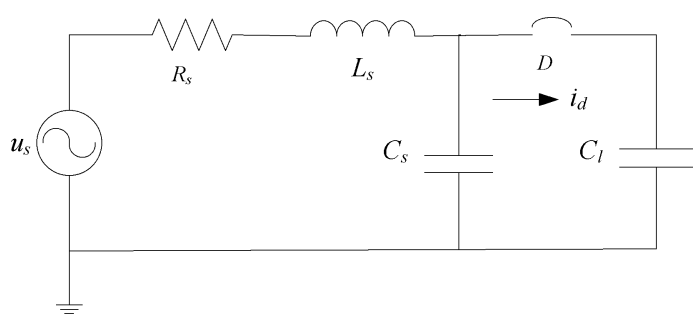

Fig. 1. Basic circuit diagram for capacitive current interruption with a disconnector.

It is assumed that the impedances $R_{s}$ and $\omega_{p} L_{s}$ are much smaller than $1 /\left(\omega_{p} C_{s, l}\right)$, meaning that the voltage $u_{c p}$ is very close to $u_{s}$.

When the disconnector opens, the interruption process begins. The basic circuit in Fig. 1 is separated into two parts abruptly. The left part of the circuit, consisting of $R_{s}, L_{s}, C_{s}$, remains energized with $u_{s}$. The voltage across $C_{s}$, denoted as $u_{c s}$, remains very close to the source $u_{s}$. The right part of the circuit only contains $C_{l}$ which has no discharge path and the voltage $u_{c l}$ across $C_{l}$ is dc due to the trapped charge. The Transient Recovery Voltage (TRV), i.e., the difference between $u_{c s}$ and $u_{c l}$ [16], and the dielectric withstand capability of the air gap between the two contacts of the disconnector are denoted as $u_{d}$ and $u_{r}$, respectively. After the contacts of the disconnector separate, the TRV starts to rise and the dielectric strength starts to recover simultaneously. Once $u_{d}$ exceeds the dielectric strength of the gap $u_{r}$, the arc re-ignites. At a sufficiently low current, the arc lasts no longer than a half power frequency cycle and extinguishes when the arc current passes through zero. When the arc extincts temporary at $i_{d}=0$, the circuit is separated into two parts again until the next reignition occurs. The interruption process may therefore be described as a periodic arc extinction and reignition. Finally, this sequence comes to an end and the arc extinguishes completely when the distance between the disconnector contacts becomes sufficiently large to prevent any further reignitions.

At each reignition, the voltages $u_{c s}, u_{c l}, u_{d}$ and current $i_{d}$ have oscillations at distinct frequencies. A high-frequency $(\mathrm{HF})$ component arises after reignition when the voltages across load- and source side capacitance are equalizing. After this HF process, the voltages $u_{c l}, u_{c s}$ change and a voltage drop arises across $L_{s}$ which causes a medium frequency (MF) oscillation in the circuit. As the HF and MF oscillations are damped out, the power frequency (PF) remains. These three components are analyzed in detail below.

\section{A. High Frequency $(\mathrm{HF})$ Component}

At the instant of reignition, the voltage $u_{c s}$ and $u_{c l}$ will equalize through a high frequency oscillation. The equalization voltage $U_{E}$ is calculated from charge conservation in both capacitances [12]

$$
U_{E}=\frac{U_{c s} C_{s}+U_{c l} C_{l}}{C_{s}+C_{l}}
$$

where $U_{c s}, U_{c l}$ are the initial voltages across $C_{s}, C_{l}$, respectively. 


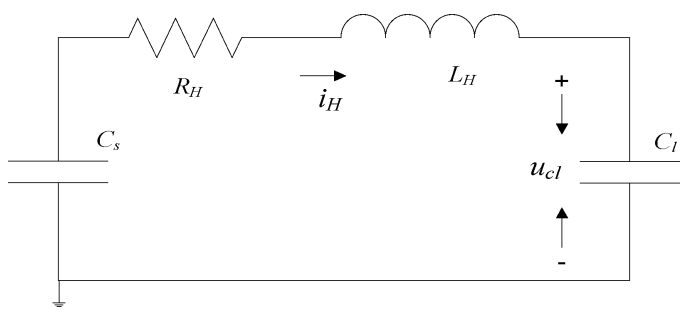

Fig. 2. High frequency equivalent circuit diagram.

In the HF circuit model shown in Fig. 2, the power supply is ignored since it is decoupled by the high impedance of inductance $L_{s}$ for the HF oscillation. $R_{H}, L_{H}$ represents the high-frequency resistive losses and inductance of the circuit formed by the capacitors, the disconnector and the arc. In Fig. 2, $u_{c l}$ and $i_{H}$ denote the voltage across $C_{l}$ and the current in the high frequency loop circuit, respectively.

The HF phenomenon lasts only a short duration (typically less than a few tens of microseconds), during which it can lead to a high transient current through the circuit and transient voltages across both capacitances.

The reignition voltage across the air gap at the reigniting time $t=0$ is defined as

$$
U_{r}=U_{c s}-U_{c l}
$$

Assuming the total resistive losses

$$
R_{H}<2 \sqrt{\left(L_{H} / C_{H}\right)},\left(1 / C_{H}\right)=\left(1 / C_{s}\right)+\left(1 / C_{l}\right),
$$

we get

$$
\left\{\begin{array}{l}
u_{c l}=U_{E}-\frac{U_{r}}{1+C_{l} / C_{s}} \frac{\omega_{0 H}}{\omega_{H}} e^{-\delta_{H} t} \sin \left(\omega_{H} t+\beta_{H}\right) \\
i_{H}=\frac{U_{r}}{L_{H} \omega_{H}} e^{-\delta_{H} t} \sin \left(\omega_{H} t\right)
\end{array} .\right.
$$

Similarly, the voltage $u_{c s}$ across the source side capacitance at $\mathrm{HF}$ can be calculated

$$
u_{c s}=U_{E}+\frac{U_{r}}{1+C_{s} / C_{l}} \frac{\omega_{0 H}}{\omega_{H}} e^{-\delta_{H} t} \sin \left(\omega_{H} t+\beta_{H}\right)
$$

where

$$
\begin{aligned}
\delta_{H} & =\frac{R_{H}}{2 L_{H}}, \omega_{0 H}=\frac{1}{\sqrt{L_{H} C_{H}}}, \\
\omega_{H}^{2} & =\omega_{0 H}^{2}-\delta_{H}^{2}, \beta_{H}=\arctan \frac{\omega_{H}}{\delta_{H}} .
\end{aligned}
$$

The parameter $\delta_{H}$ is the damping coefficient at HF.

The HF oscillation frequency $\omega_{H}$ depends mainly on the stray inductance and the series connected value of both capacitances. This high frequency can be up to several MHz. The oscillation across the capacitances eventually ceases and settles at a quasi steady state value $U_{E}$.

According to (4) and (5), the maximum transient voltage across $C_{s}, C_{l}$ (which occurs at negligible $\mathrm{HF}$ damping) is $\left|\left(U_{r}\right) /\left(1+C_{s} / C_{l}\right)\right|+\left|U_{E}\right|$ and $\left|\left(U_{r}\right) /\left(1+C_{l} / C_{s}\right)\right|+\left|U_{E}\right|$, respectively. The maximum transient current is about $\left(U_{r} / \sqrt{L_{H} / C_{H}}\right)$ which depends on the reignition voltage $U_{r}$ and the electrical parameters of the HF loop.
It is evident that the smaller capacitance has the higher maximum voltage and this increase while $u_{r}$ increases. Further, $u_{r}$ increases with increasing contact distance, which means that HF voltages across the capacitances will become largest just before the arc extincts completely. The maximum transient voltage that can occur for HF is $3 E_{m}$, if $U_{r}=2 E_{m}$ and either $C_{s} \ll C_{l}$ or $C_{s} \gg C_{l}$. The current $i_{H}$ through the disconnector in the HF loop increases with $u_{r}$ and $C_{s}, C_{l}$. Thus $u_{r}$ and $C_{s} / C_{l}$ (or $C_{l} / C_{s}$ ) are the key parameters that affect the voltage and current behavior in the HF component of reignition.

\section{B. Medium Frequency (MF) Component}

Upon reignition, also a medium frequency oscillation starts. The MF component which lasts about a few milliseconds also causes a transient voltage and current. At this stage the voltage across the arc is neglected. $R_{H}, L_{H}$ in Fig. 2 are neglected as well, because their equivalent impedances are much smaller than the capacitance's impedance at MF. Therefore $C_{s}$ and $C_{l}$ with an identical initial voltage $U_{E}$ are in parallel and the equivalent circuit of Fig. 1 can be applied. Because of the charge redistribution during the HF part, the voltages across $C_{s}$ and $C_{l}$ have changed and cause a voltage drop across $L_{s}$ and $R_{s}$.

The time of reignition is again taken as $t=0$. On the time scale of the MF oscillation, $u_{s}$ can be treated as a constant $\left(U_{s}=\right.$ $\left.E_{m} \sin \phi\right)$. Similarly as for the HF analysis, we find for $u_{C M}$ (the voltage across $C_{s}$ and $C_{l}$ at $\mathrm{MF}$ ), and for $i_{M}$ (the current through the disconnector at MF)

$$
\left\{\begin{array}{l}
u_{C M}=U_{S}-\frac{U_{r}}{1+C_{s} / C_{l}} \frac{\omega_{0} M}{\omega_{M}} e^{-\delta_{M} t} \sin \left(\omega_{M} t+\beta_{M}\right) \\
i_{M}=\frac{1}{\left(1+C_{s} / C_{l}\right)^{2}} \frac{U_{r}}{L_{s} \omega_{M}} e^{-\delta_{M} t} \sin \left(\omega_{M} t\right)
\end{array}\right.
$$

where

$$
\begin{aligned}
& \delta_{M}=\frac{R_{S}}{2 L_{S}}, \omega_{0 M}=\sqrt{\frac{1}{L_{S}\left(C_{s}+C_{l}\right)}}, \\
& \omega_{M}^{2}=\omega_{0 M}^{2}-\delta_{M}^{2}, \beta_{M}=\arctan \frac{\omega_{M}}{\delta_{M}} .
\end{aligned}
$$

The oscillation at medium frequency $\omega_{M}$ mainly depends on both capacitances and inductance $L_{s}$. Generally speaking this oscillation frequency is in the order of several $\mathrm{kHz}$.

Similarly, as for the high frequency transient, the voltages in MF across both capacitances with initial voltages $U_{E}$ are damped due to the equivalent resistance in the loop and finally reach the value $U_{s}$. The maximum transient voltage is about $\left|U_{S}\right|+\left|\left(U_{r}\right) /\left(1+C_{s} / C_{l}\right)\right|$ and increases with increasing $U_{r}, C_{l} / C_{s}$ and $U_{s}$. Similar to the HF analysis, the maximum theoretical transient voltage is $3 E_{m}$. The current through the disconnector at MF depends on $U_{r}$, capacitance ratio and $L_{s}$. The maximum current also increases with increasing $U_{r}$.

\section{Three Components Synthesis}

After the HF and MF components have damped out, only the PF component remains. Since the time constants involved differ considerably, the HF component has disappeared on the timescale for MF and MF has disappeared on the timescale for $\mathrm{PF}$. For instance, the initial voltage at MF is the steady state voltage at $\mathrm{HF}$ and the initial voltage at $\mathrm{PF}$ is the steady state voltage at MF. In order to find the complete behavior, the three 
components are combined. The voltage $u_{c l}$ across the load side capacitance and the current $i_{d}$ flowing through the disconnector at reignition can be written as

$$
\begin{aligned}
u_{c l}= & -\frac{U_{r}}{1+C_{l} / C_{s}} \frac{\omega_{0 H}}{\omega_{H}} e^{-\delta_{H} t} \sin \left(\omega_{H} t+\beta_{H}\right) \\
- & \frac{U_{r}}{1+C_{s} / C_{l}} \frac{\omega_{0 M}}{\omega_{M}} e^{-\delta_{M} t} \sin \left(\omega_{M} t+\beta_{M}\right) \\
& +E_{m} \sin \left(\omega_{P} t+\phi\right) \\
i_{d}= & \frac{U_{r}}{L_{H} \omega_{H}} e^{-\delta_{H} t} \sin \left(\omega_{H} t\right) \\
& +\frac{1}{\left(1+C_{s} / C_{l}\right)^{2}} \frac{U_{r}}{L_{s} \omega_{M}} e^{-\delta_{M} t} \sin \left(\omega_{M} t\right) \\
& +C_{l} \omega_{P} E_{m} \cos \left(\omega_{P} t+\phi\right) .
\end{aligned}
$$

Equations (7) and (8) are the combination of the three frequency components in the voltage across the load side capacitance and the current through the disconnector on reignition respectively. The voltage across the source side capacitance can be calculated in a similar manner.

Equations (7) and (8) show that the voltage and current in the loop, on reignition, depend on the air gap reignition voltage, power supply level and the network electrical parameters. In order to further understand the capacitive current interruption with an air-break disconnector, Section III focuses on this phenomenon experimentally.

\section{EXPERIMENTAL RESULTS INVESTIGATION}

A series of tests is performed on capacitive current interruption with an air-break high-voltage disconnector in a test-circuit. Those measurements are carried out at $90 \mathrm{kV}$ to $173 \mathrm{kV}$ supply voltage in the KEMA High Power Laboratory, the Netherlands. The basic simplified test circuit is shown in Fig. 1. The current to be interrupted varies over a range from $0.23 \mathrm{~A}$ to $2.1 \mathrm{~A}$, and the source- and load side capacitance are taken in the range of $C_{s}=1.5 \mathrm{nF}-100 \mathrm{nF}, C_{l}=4.3 \mathrm{nF}-40 \mathrm{nF}$ respectively. Various combinations of current and of $C_{s}$ and $C_{l}$ are selected. The value of $L_{s}$ is fixed at $480 \mathrm{mH}$. A center-break type disconnector with rated voltage of $300 \mathrm{kV}$ is subjected to the test. The bandwidth of the voltage divider and the current transformers allows to measure signals up to $1 \mathrm{MHz}$ and $100 \mathrm{kHz}$, respectively. Noise with frequencies above the bandwidths is filtered from the recorded signals.

During the experiments, general interruption characteristics such as arc duration, gap length, the blade angle at arc extinction, and overvoltage across $C_{l}$ are recorded. The instantaneous current $i_{d}$, the voltages $u_{c s}$ and $u_{c l}$ are recorded as well. Further, high-speed video recording of the arc is made. The initial analysis of the measured data has been done in [12] and [13] and revealed as follows.

- Arc duration increases with magnitude of current interrupted (at constant $C_{s}$ ), and also increases with decreasing value of $C_{s} / C_{l}$.

- The minimum blade angle of the disconnector required for the arc extinction is about 50 degrees. The disconnector can be close to fully open for the smallest values of $C_{s} / C_{l}$ before the current was finally interrupted.
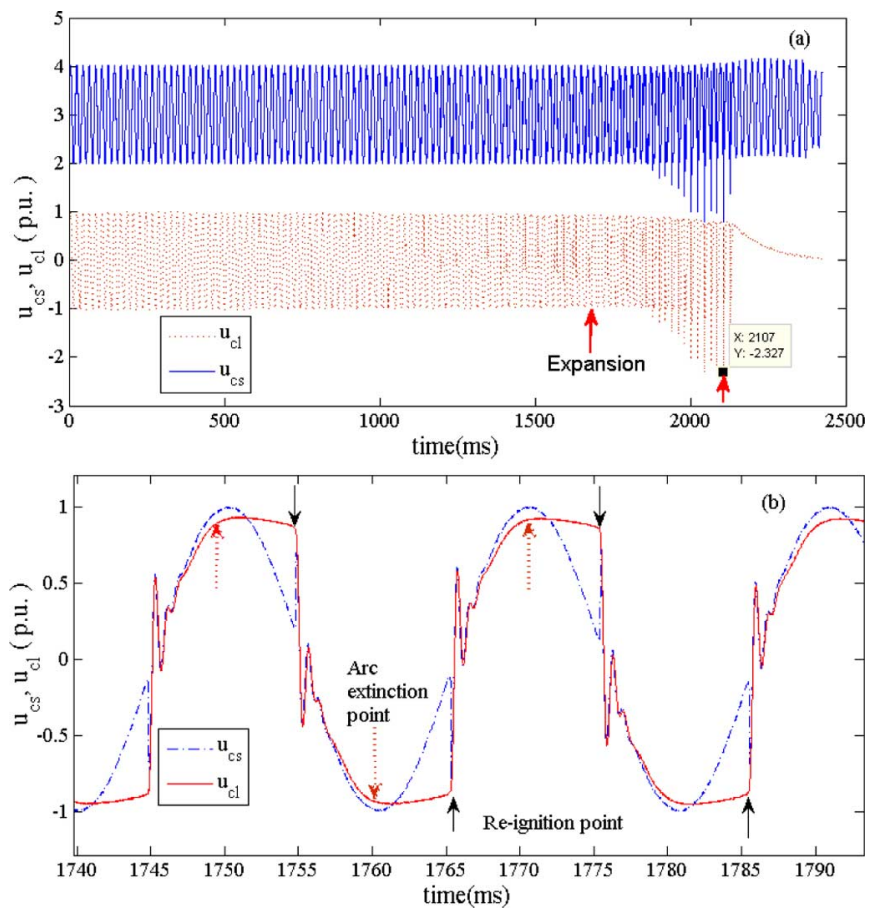

Fig. 3. Typical wave shapes of (a) voltage $u_{c s}, u_{c l}$ and (b) their expansions.

- The overvoltage across the load side capacitor reached a maximum value of 2.33 p.u. when $C_{s} / C_{l} \ll 1$.

- The thermal effect which affects the arc behavior becomes significant for currents larger than $1 \mathrm{~A}$.

Most of these conclusions can be explained by the theoretical model mentioned in Section II, showing that with smaller $C_{s} / C_{l}$, the transients in current and voltage are larger especially at MF.

In the following section, a more detailed analysis of the measured data is given. First, the various typical wave shapes are shown of the relevant transient phenomena during arcing. Second, the interruption process is analyzed, taking into account the reignition voltage and the energy input to the arc during the reignitions.

\section{A. Voltage and Current Wave Shapes From Measurements}

During the tests two high voltage dividers are used to measure voltage across $C_{s}$ and $C_{l}$. Typical waveforms of $u_{c s}, u_{c l}, i_{d}, u_{d}$ are shown in Figs. 3-5. The circuit parameters for this measurement are: $U_{s}=173 \mathrm{kV} \mathrm{rms}, C_{s}=1.5 \mathrm{nF}, C_{l}=40 \mathrm{nF}$. The waveforms of Figs. 3-5 confirm that the capacitive current interruption with an open-air disconnector consists of multiple arc reignitions and arc extinctions. The arc extincts (temporarily) regularly at the arc current zero at each half cycle. This means that the reignition of the arc mainly depends on TRV and capability of the air gap to withstand it.

During the interruption, the maximum overvoltage of $u_{c l}$ is 2.33 p.u [see Fig. 3(a)]. The maximum MF transient current of about $60 \mathrm{~A}$ [see Fig. 5(a)] is observed (the measuring system does not allow observation of the HF current component). Within the chosen test parameters the overvoltage became largest just before complete arc extinction, which is in agreement with the theoretical analysis in Section II [see Fig. 3(a)]. 

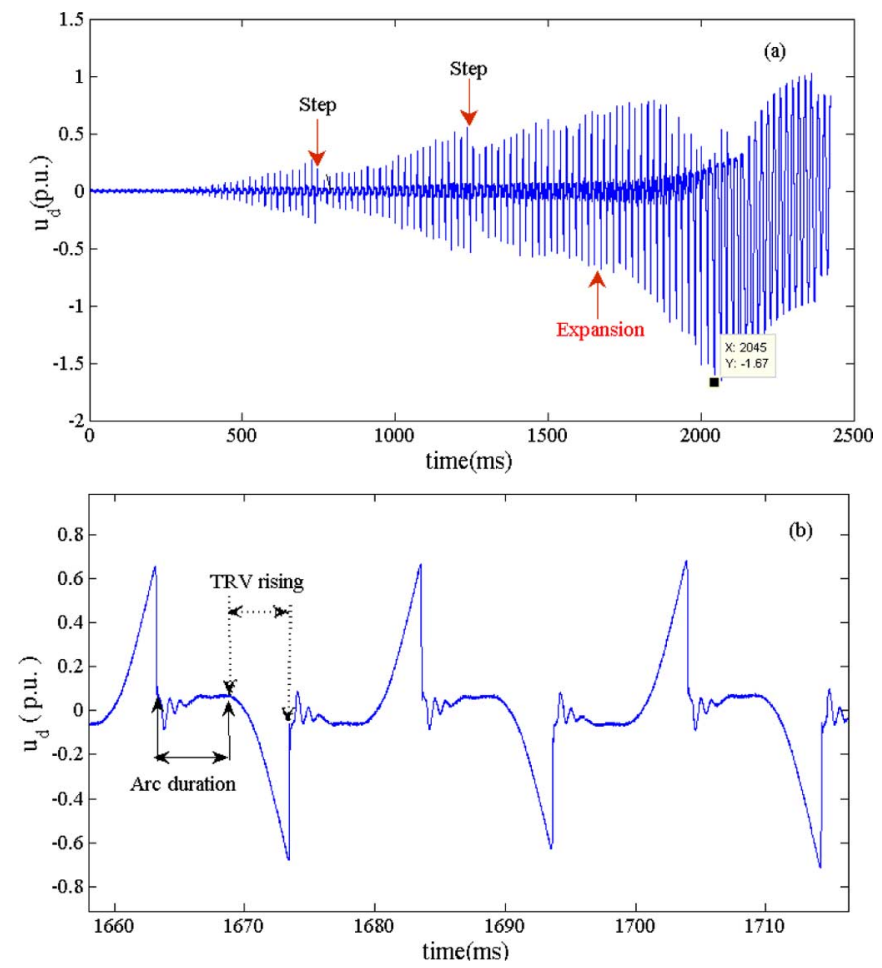

Fig. 4. Typical wave shapes of (a) voltage $u_{d}$ and (b) its expansion.
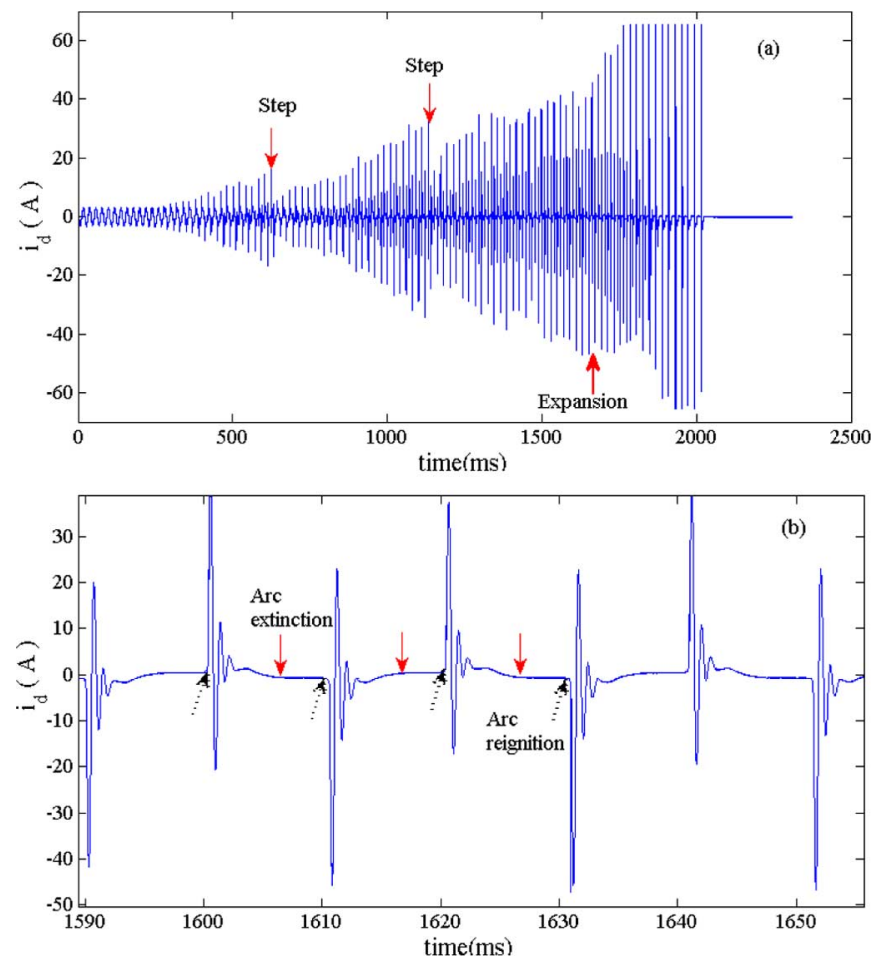

Fig. 5. Typical wave shapes of (a) current $i_{d}$ and (b) its expansion.

The voltage $u_{d}$ across the disconnector is not measured directly, but is determined as the difference between voltages $u_{c s}$ and $u_{c l}$. The absolute error in the dividers is with a few percent too high for determining the arc voltage by subtracting these voltages directly. Therefore a correction is made by adjusting the measured voltages such that they become equal with closed disconnector. The typical $u_{d}$ waveform of Fig. 4 clearly shows the arc duration and Transient Recovery Voltage (TRV) rising at each half power frequency cycle.

An interesting feature is that values of $u_{d}, i_{d}$ (Figs. 4 and 5) on each moment of reignition do not rise continuously with the increasing contacts distance, but a few "steps" are observed. This phenomenon indicates that reignition voltage not only is determined by the distance between two contacts of the disconnector but also depends on other influences, the most important of which is a reduction of breakdown voltage due to the heating of the air by the arc.

\section{B. Reignition Voltage}

By analyzing the disconnector voltage $u_{d}$, the reignition voltage $\left(u_{r}\right)$ development can be obtained. In order to present the reignition voltage waveshapes, one group of measured data is selected. The results for the measurements performed at a power supply level of $173 \mathrm{kV}$ are given in Fig. 6. The following observations are made:

- Reignition voltage level can be as high as $500 \mathrm{kV}(2.05$ p.u.) at $C_{s} / C_{l}=3.1$. It does not increase continuously but with a few "steps" at both positive and negative polarities.

- The current $i_{d}$ and the ratio of $C_{s} / C_{l}$ significantly influence the reignition voltage and arc duration. The reignition voltage increases with decreasing $i_{d}$ and increasing $C_{s} / C_{l}$. The reason is that with larger $i_{d}$, and smaller $C_{s} / C_{l}$, there is a higher energy input into the arc on reignition. The arc path needs more time to recover its dielectric strength.

- The positive and negative reignition voltages are not symmetrical. For example, at 2.1 A in Fig. 6(a) the negative voltage is larger than the positive reignition voltage, especially close to the final arc extinction point; at $C_{s} / C_{l}=$ 0.08 in Fig. 6(b), the positive reignition voltage is larger than the negative reignition voltage as well.

- As mentioned before, a reignition occurs each half power frequency period. However, because of the polarity dependence which causes an asymmetrical overvoltage across $C_{s}$ and $C_{l}$, a few groups of measured data show only one reignition within each full power frequency cycle. Further, the experimental results show that this phenomenon only happens at $C_{s} / C_{l}<0.1$.

\section{Energy Input into the Arc on Reignition}

The energy input into the arc on reignition is an important influential factor for the arc duration, and the next reignition voltage value. Once a reignition occurs, the arc connects two capacitances electrically. There is a current $i_{d}$ through the arc and a voltage $u_{d}$ across the arc. The energy input into the arc is calculated by integration in each half cycle the product of the current $i_{d}$ and voltage $u_{d}$ starting from the moment of reignition $t_{1}$ until the (temporary) arc extinction $t_{2}: E_{\text {arc }}=\int_{t_{1}}^{t_{2}} u_{d} i_{d} d t$. From the typical energy waveshapes shown in Figs. 7 and 8, the following conclusions are given:

- The energy input into the arc on reignition is typically a few hundred Joule and up to a few thousands Joule at lower ratio of $C_{s} / C_{l}$. It becomes larger gradually (with occasional "steps") when the contacts of disconnector are 

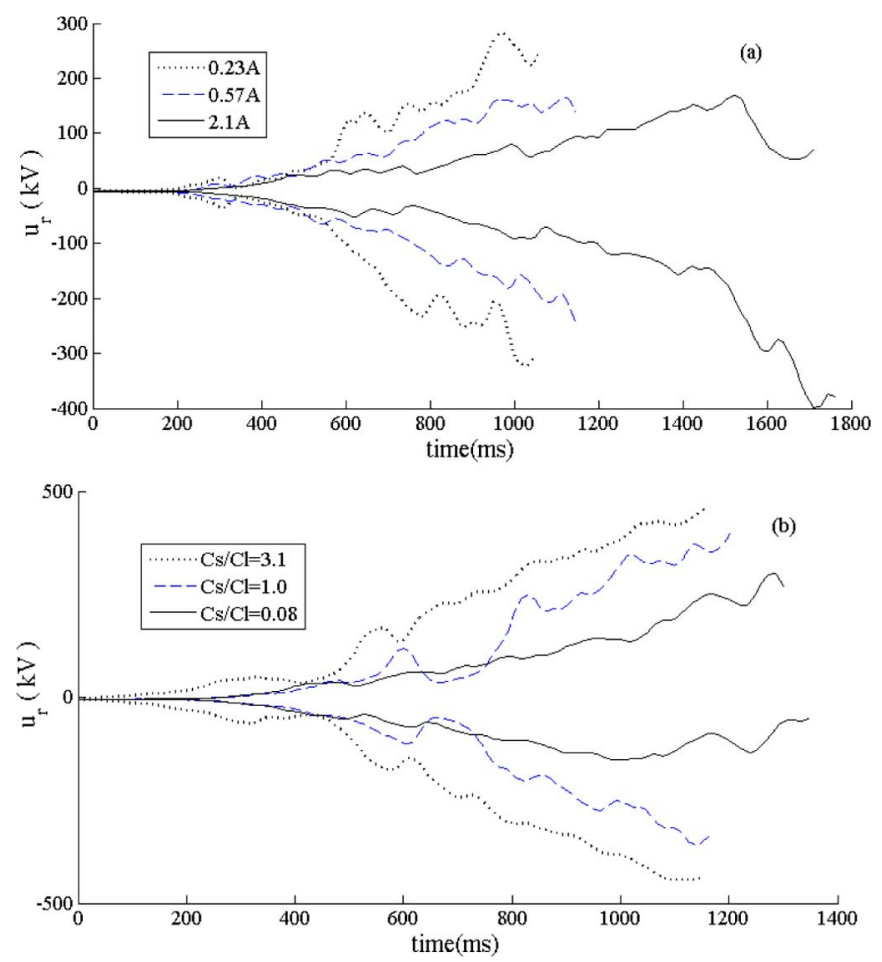

Fig. 6. Effects of current and capacitor ratio $C_{s} / C_{l}$ on reignition voltage (a) $i_{d}$ with $C_{s}=1.5 \mathrm{nF}$, (b) $C_{s} / C_{l}$ with $i_{d}=2.1 \mathrm{~A}$.

moving away. It reaches the largest value, just before complete arc extinction.

- The energy level at each half cycle rises very fast once the reignition starts, where the higher frequencies components dominate. Then it remains almost constant during the power frequency period (also see Fig. 7) [14].

- $C_{s}, C_{l}, i_{d}$ have significant influence on the arc energy and on the reignition probability. It can be observed that the energy input into the arc is higher with higher interruption current and lower $C_{s}$.

\section{Comparison With Theoretical Analyses}

The bandwidth and sampling rate of the measuring system in the test are too low to show the entire HF component. Only the medium frequency component therefore can be compared with theory in detail in this contribution.

First, a specific measurement with one group of parameters is discussed. In order to compare with measured results, the parameters selected are as close as possible to those in the real test: $U_{r}=410 \mathrm{kV}$ (see Fig. 4), $C_{s}=1.5 \mathrm{nF}, C_{l}=40 \mathrm{nF}, L_{s}=480$ $\mathrm{mH}, R_{s}=3 \mathrm{k} \Omega, R_{H}=25 \Omega, L_{H}=15 \mu \mathrm{H}, E_{m}=173 \times \sqrt{ } 2$ $\mathrm{kV}, \phi=90^{\circ}$.

The simulated waveshape for the voltage across the load side capacitance on the reignition is shown in Fig. 9. It shows clearly that $u_{c l}$ has three components $\mathrm{HF}, \mathrm{MF}$, and PF and there are oscillations at HF and MF. The HF component lasts a few microseconds and the MF component lasts a few milliseconds. The MF component, which lasts about $4 \mathrm{~ms}$ according to the model in Fig. 9, has the same duration as in the measurement (see Figs. 3-5). The medium frequency which is $1 \mathrm{kHz}$ by theory
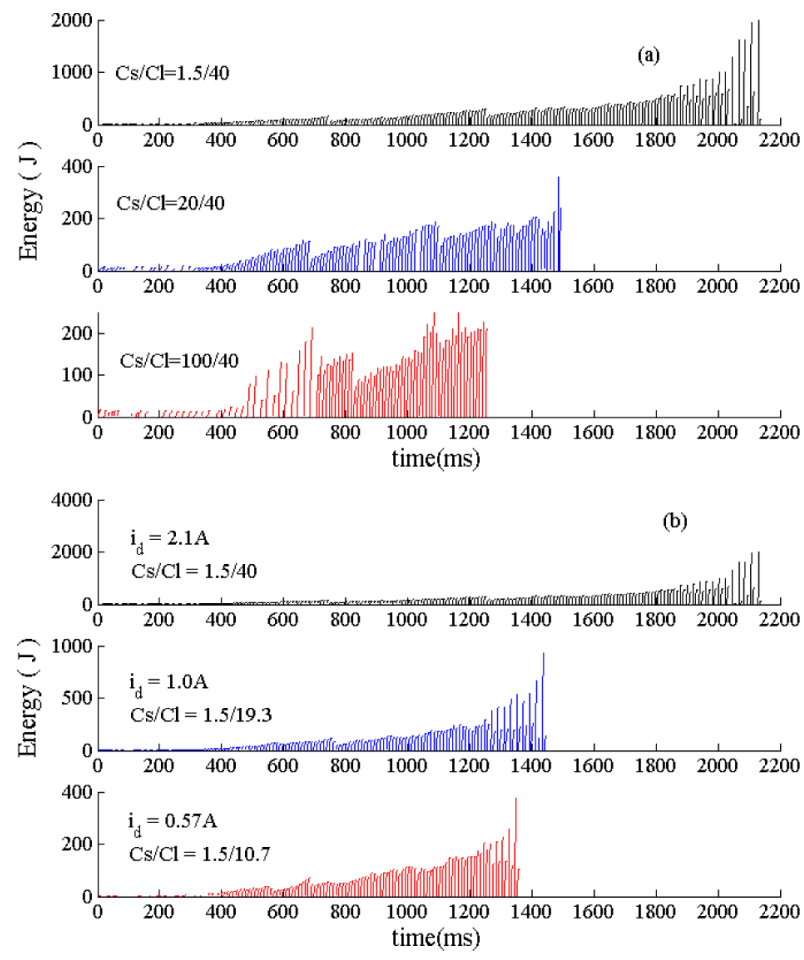

Fig. 7. Energy input into the arc on reignition versus time with parameters current and capacitor ratio $C_{s} / C_{l}$. (a) $C_{s} / C_{l}\left(i_{d}=2.1 \mathrm{~A}\right)$. (b) $i_{d}\left(C_{s}=\right.$ $1.5 \mathrm{nF})$.

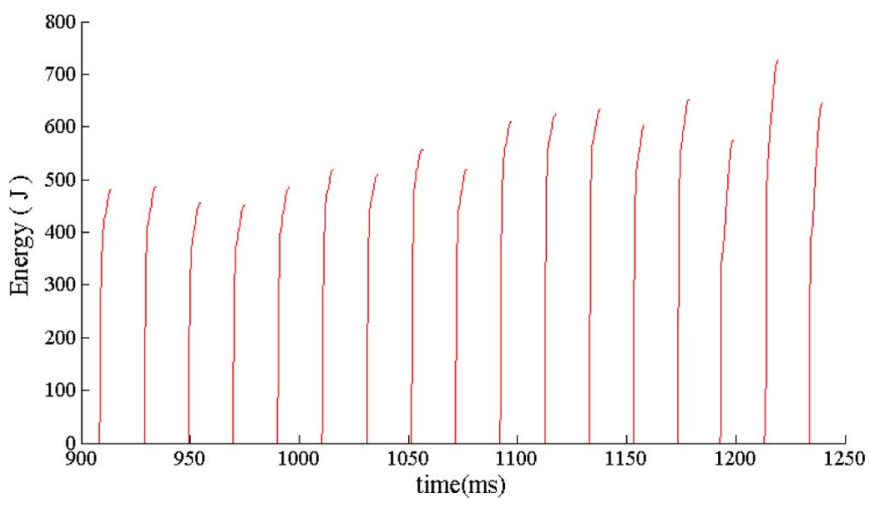

Fig. 8. Expansion at $\mathrm{t}=900-1260 \mathrm{~ms}$ from Fig. 7(a) with $C_{s} / C_{l}=$ $100 / 40$

is $963 \mathrm{~Hz}$ in the measurement. Therefore, for the medium frequency phenomena measurement and theory are in agreement.

Second, the measured and calculated overvoltages across load side capacitance are compared in detail below. Table I shows that the calculated results are slightly larger than those obtained from measurement, probably because of differences in damping. The predicted maximum overvoltage of 3 p.u. is higher than the value of 2.33 p.u. observed in the measurements.

The data analyzed from measurement show that the transients during interruption are qualitatively in agreement with the theoretical analysis.

\section{IMPLICATIONS FOR STANDARDIZATION}

As already pointed out in Sections II and III, the macroscopic arc behavior is strongly dependent on the circuit as quantified as 

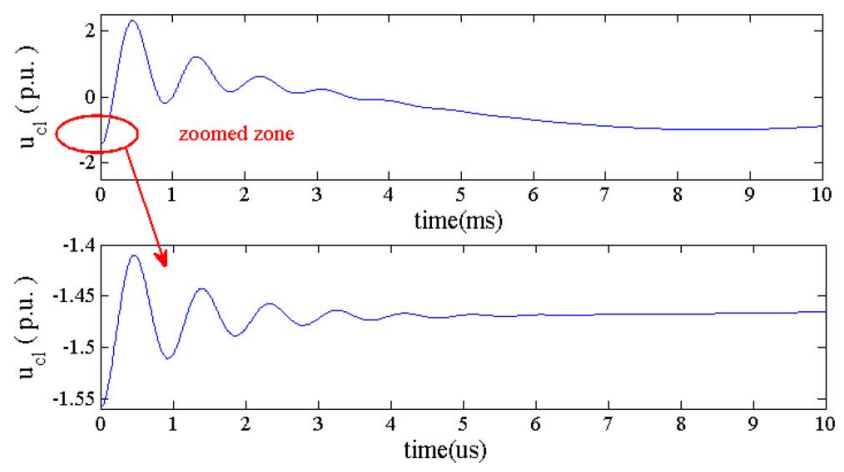

Fig. 9. Waveshape for overvoltage across load side capacitance and its HF expansion.

TABLE I

Compared Overvoltage BetweEn Measurement RESULTS AND CALCULATION

\begin{tabular}{|c|c|c|c|}
\hline$u_{s}(\mathrm{kV})$ & $C_{\mathcal{S} / C_{l}}$ & $u_{c l}$ (p.u.) & $u_{c l}^{\prime}$ (p.u.) \\
\hline 90.0 & $60 / 19.3$ & 1.39 & 1.52 \\
\hline 171.5 & $6 / 19.3$ & 2.09 & 2.32 \\
\hline 173.0 & $1.5 / 40$ & 2.33 & 2.57 \\
\hline 173.0 & $6 / 40$ & 2.25 & 2.40 \\
\hline
\end{tabular}

Note: $u_{c l}$ (p.u.), $u_{c l}^{\prime}$ (p.u.) is overvoltage across load side capacitance from measurement and calculation respectively

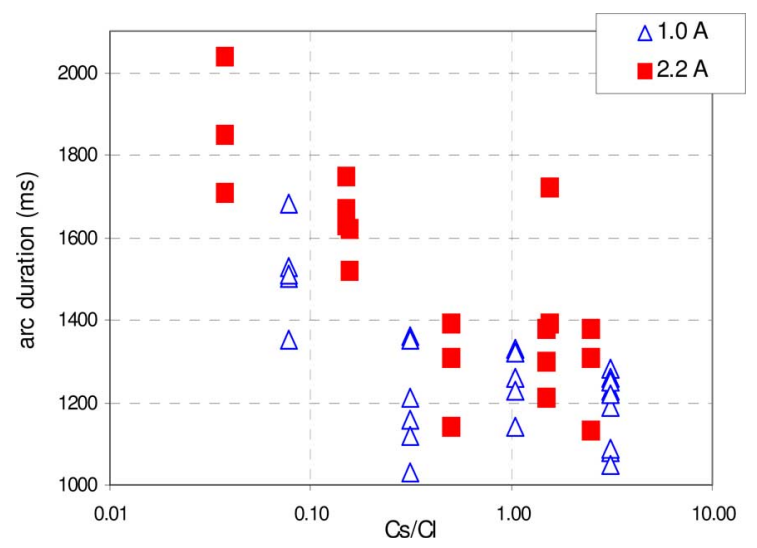

Fig. 10. Disconnector arc duration as a function of $C_{s} / C_{l}$ for arc current 1.0 and $2.2 \mathrm{~A}$.

$C_{s} / C_{l}$. This is also illustrated in Figs. 10 and 11 showing the arc duration and observed overvoltages as a function of $C_{s} / C_{l}$ for two values of the current.

This observation implies that for testing of the disconnector switching capability, the circuit plays a major role (this also applies to the testing of auxiliary interrupting devices such as so-called whips). Since no test-circuit has been defined yet, one of the tasks of the IEC maintenance team, elaborating an amendment to the IEC standard 62271-102 [1] was to define a circuit. It was decided that $20 \mathrm{CO}$ (close-open) tests have to performed with $C_{s} / C_{l}=0.1$, adopting a test-circuit as in Fig. 1. Alternative, but yet adequate supply circuits supplying much less than the short-time current are discussed. It was decided to give the document the status of a technical report and allow time for collecting experience. The technical report will be issued in 2009 [17].

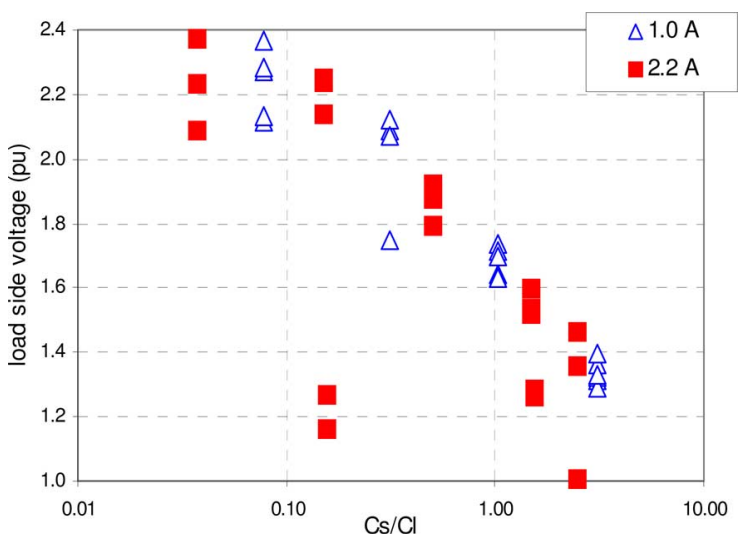

Fig. 11. Measured overvoltages (p.u.) across load as a function of $C_{s} / C_{l}$.

\section{CONCLUSION}

In this paper, in order to investigate small capacitive current interruption phenomena with a high-voltage air-break disconnector, a relative simple circuit is selected for study from theoretical and experimental point of view.

Both approaches show that the capacitive current interruption with an air-break disconnector is an event with multiple reignitions containing high frequency (a few $\mathrm{MHz}$ ), medium frequency (a few $\mathrm{kHz}$ ) and power frequency components in current and voltage. These reignitions can cause significant overvoltages (a value up to 2.33 p.u. was experimentally observed whereas 3 p.u. was predicted as a theoretical maximum), higher transient currents and prolong arc duration. This makes the interruption more severe and might cause damage to nearby equipment.

Specifically, the energy input to the arc, the overvoltage, the (transient) current depend on the interrupted current $i_{d}, C_{s}, C_{l}$ (especially the ratio $C_{s} / C_{l}$ ) and source voltage $u_{s}$. At lower values of $C_{s} / C_{l}$ (with ratio less than one), higher current $i_{d}$, higher voltage power supply $u_{s}$, the arc duration and overvoltage across the load tend to increase. The results show that by a suitable choice of $C_{s} / C_{l}$, arc duration and transient voltage and current may be reduced. Making $C_{s} / C_{l}$ as large as possible is one option. Larger $C_{s} / C_{l}$ leads to a lower energy input into the arc and makes the air gap to recover its dielectric strength faster. It is therefore easier to interrupt with shorter arcs and with lower overvoltages.

Also, the energy input into the arc, the overvoltages and the transient current in the circuit always reach the largest value just before the arc extincts completely. Reducing the arcing time (making the arc extinct with lower reignition voltage at the end) therefore is a key problem to be solved for this issue.

The final purpose of this project is to develop air break disconnectors that have increased current interruption capability.

\section{REFERENCES}

[1] IEC Standard for High-voltage Switchgear and Control Gear-Part 102: Alternating Current Disconnectors and Earthing Switches, IEC 62271-102, Dec. 2001.

[2] P. A. Abetti, "Arc interruption with disconnecting switches," M.Sc. dissertation, Illinois Inst. Technol., Chicago, IL, Jan. 1948.

[3] F. E. Andrews, L. R. Janes, and M. A. Anderson, "Interrupting ability of horn-gap switches," AIEE Trans., vol. 69, pp. 1016-1027, Apr. 1950. 
[4] E. C. Rankin, "Experience with methods of extending the capability of high-voltage air break switches," AIEE Trans. Power App. Syst., vol. 78, pp. 1634-1636, Dec. 1959.

[5] A. Foti and J. M. Lakas, "EHV switch tests and switching surges," IEEE Trans. Power App. Syst., vol. 83, pp. 266-271, Mar. 1964.

[6] IEEE Committee Rep., "Results of survey on interrupting ability of air break switches," IEEE Trans. Power App. Syst., vol. PAS-85, no. 9, pp. 1008-1019, Sep. 1966.

[7] Canadian Electrical Association, "The interrupting capability of high voltage disconnect switches," CEA Project 069 T 102 rep., Jul. 1982.

[8] D. F. Peelo, "Current interrupting capability of air break disconnect switches," IEEE Trans. Power Del., vol. 1, no. 1, pp. 212-216, Jan. 1986.

[9] S. G. Patel, W. F. Holcombe, and D. E. Parr, "Application of air-break switches for de-energizing transmission lines," IEEE Trans. Power App. Syst., vol. 4, no. 1, pp. 368-374, Jan. 1987.

[10] H. Knobloch, "Switching of capacitive currents by outdoor disconnectors," presented at the 5th Int. Symp. High Voltage Engineering, Braunschweig, Germany, Aug. 1967.

[11] IEEE Guide to Current Interruption With Horn-Gap Air Switches, IEEE Std. C37.36b-1990, Jul. 1990

[12] D. F. Peelo, "Current interruption using high voltage air-break disconnectors," Ph.D. dissertation, Dept. Elect. Eng., Eindhoven Univ. Technol., Eindhoven, The Netherlands, 2004.

[13] D. F. Peelo, R. P. P. Smeets, L. van Der Sluis, S. Kuivenhoven, J. G. Krone, J. H. Sawada, and B. R. Sunga, "Current interruption with high voltage air-break disconnectors," in Proc. CIGRE Conf., Paris, France, 2004, paper A3-301.

[14] D. F. Peelo, R. P. P. Smeets, and J. G. Krone, "Capacitive current interruption in atmospheric air," in Proc. 2005 CIGRE A3/B3 Colloquium Conf., Tokyo, Japan, paper no. 106.

[15] S. Carsimamovic, Z. Bajramovic, M. Ljevak, M. Veledar, and N. Halilhodzic, "Current switching with high voltage air disconnector," presented at the Int. Conf. Power Systems Transients Conf., Montreal, QC, Canada, 2005, paper no. IPST05-229.

[16] L. van der Sluis, Transients in Power Systems. New York: Wiley, 2001, p. 49.

[17] "Capacitive current switching capability of air-insulated disconnectors," IEC Tech. Rep. 62271-304, 2009, to be issued.

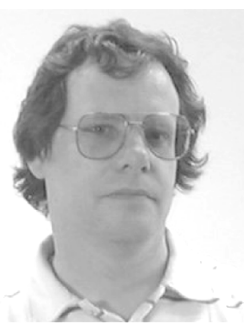

P. A. A. F. Wouters was born in Eindhoven, the Netherlands, on June 9, 1957. He received the Ph.D. degree in elementary electronic transitions between metal surfaces and low energetic (multiple) charged ions the Utrecht University (UU), Utrecht, the Netherlands, in 1989.

In 1990, he joined the Electrical Power Systems (EPS) group at the Eindhoven University of Technology, Eindhoven, the Netherlands, as Research Associate. His research interests include partial discharge techniques, vacuum insulation, and LF electromagnetic-field screening. Currently, he is Assistant Professor in the field of diagnostic techniques in high-voltage systems.

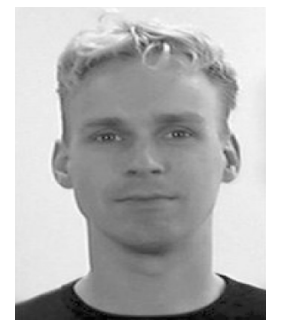

R. T. W. J. van Hoppe is involved with activities at the Eindhoven University of Technology, Eindhoven, The Netherlands, for guiding/supporting students (graduating/Ph.D.) during their training, research, and/or learning processes. He is a Lecturer/Instructor for some courses.

He is involved as an expert in a student educational project. The other part involves work in research projects regarding high voltage, pulsed power, and electromagnetic compatibility. He began with the Electrical Power Systems Group at the Eindhoven University of Technology in 2001.

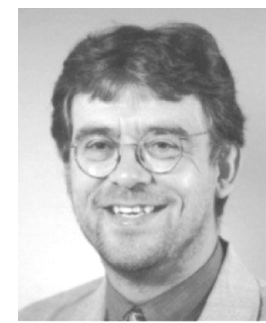

R.P.P. Smeets (F'08) was born in 1955 . He received the M.Sc. degree in physics from the Eindhoven University of Technology, Eindhoven, The Netherlands, in 1981 .

$\mathrm{He}$ received the $\mathrm{Ph} . \mathrm{D}$. degree in research work on switching arcs in 1987. He was an Assistant Professor with Eindhoven University, Eindhoven, The Netherlands, until 1985. In 1991, he was with Toshiba Corporation's Heavy Apparatus Engineering Laboratory, Japan. In 1995, he joined KEMA T\&D Testing Services. Currently, he manages the R\&D activities of KEMA's High Power Laboratory. In 2001, he was appointed Part-Time Professor at the Eindhoven University of Technology. He is/has been chairman and member of various IEC and CIGRE study groups. He is chairman of the "Current Zero Club". He published many papers on high-power switching and testing in international magazines and conference proceedings.

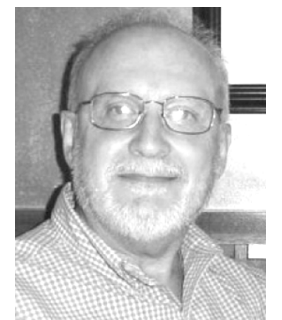

D. F. Peelo (SM'91) is an independent consultant. He was with BC Hydro, BC, Canada, for 28 years, rising to the position of Specialist Engineer, Switchgear and Switching. He was also with ASEA, Sweden, for seven years before joining BC Hydro. He has published many papers on switching and metal-oxide surge arrester application and is active in leadership roles with IEEE, CIGRE, and IEC

$\mathrm{He}$ received the Ph.D. degree for original research on current interruption using air-break disconnecting switches from the Eindhoven University of Technology, Eindhoven, The Netherlands. He is convener of IEC Maintenance Team 32 Inductive Load Switching and Maintenance Team 42 Capacitive Current Switching Capability of Disconnectors. 